\title{
Author Correction: A power-law decay evolution scenario for polluted single white dwarfs
}

Di-Chang Chen (D), Ji-Lin Zhou (D), Ji-Wei Xie (D), Ming Yang (D), Hui Zhang, Hui-Gen Liu, En-Si Liang, Zhou-Yi Yu and Jia-Yi Yang

Correction to: Nature Astronomy https://doi.org/10.1038/s41550-018-0609-7, published online 5 November 2018.

The version of this Letter originally published contained various errors in the reference list, all of which have now been corrected:

Refs 5, 6, 28, 36, 52 and 53 showed a page range instead of an article number.

Refs 9, 36 and 45 had typos in author names.

Refs 12, 24 and 49 had incorrect journal names.

Refs 17 and 53 had incorrect volume numbers.

Ref. 28 had an incorrect year.

Refs 14,19 and 47 were citations to the wrong articles.

The reference list in the originally published Supplementary Information file also contained various errors, all of which have now been corrected:

Refs $1,6,9,15,16,17$ and 32 showed a page range instead of an article number.

Ref. 1 had an incorrect year.

Ref. 10 was a citation to the wrong article.

Ref. 23 showed an article number instead of a page range.

Published online: 19 November 2018

https://doi.org/10.1038/s41550-018-0647-1 